\title{
Programa de atividade física melhora aspectos da depressão e aptidão física de dependentes químicos
}

\author{
Physical activity program improves aspects of depression physical fitness of \\ chemical dependents
}

Marciel José da Silva ${ }^{1}$, Péricles Soares Bernardes ${ }^{2}$, Amanda Rodrigues Borges ${ }^{1}$, Viviane Soares $^{1 *}$

ARTIGO ORIGINAL | ORIGINAL ARTICLE

\begin{abstract}
Este estudo teve como objetivo verificar os efeitos da atividade física em caráter de lazer sobre os sintomas de ansiedade e da aptidão física de dependentes químicos e, analisou também algumas valências físicas como promoção de saúde. Por meio de um estudo de campo de carater experimental sem grupo de controle no Centro de Reabilitação e Amparo Bom Jardim - Distrito de Interlândia - Anápolis - Goiás. Participaram do estudo 8 internos adultos, homens, em tratamento voluntário na unidade. Foram realizadas 12 intervenções. Após uma abordagem inicial foi aplicado o questionário de ansiedade e depressão de BECK e os testes de aptidão física antes e depois das intervenções. Após a intervenção, os participantes realizaram maior número de flexões de membro superior $(\mathrm{p}=0,03)$ e percorreu maior distância $(200 \mathrm{~m})$ no teste de aptidão cardiorrespiratória $(\mathrm{p}=0,002)$. Foi constado através do inventario de depressão de BECK que 4 internos se encontravam em estado de depressão mínima e 4 apresentavam score moderado de depressão. Com a intervenção foi reduzido o escore de 9 (5) para 3 (3) $(\mathrm{p}<0,001)$

Palavras-chave: usuários de droga, ansiedade, depressão, exercício.
\end{abstract}

ABSTRACT

The aim of this study was to verify the effects of leisure time physical activity on the symptoms of anxiety and the body fitness of chemical dependents and analyzed some physical variables such as health promotion. By means of experimental study without control group in the Center of Rehabilitation and Amparo Bom Jardim - District of Interland - Anápolis - Goiás. Participated in the study 8 male adults, in voluntary treatment in the unit. Twelve interventions were performed. After an initial of intervention, the BECK anxiety and depression questionnaire and the physical fitness tests were applied before and after the. After the intervention, the participants performed a higher number of upper limb flexions $(p=0.03)$ and ran a greater distance $(200 \mathrm{~m})$ in the cardiorespiratory fitness test $(\mathrm{p}=0.002)$. It was recorded through the inventory of depression of BECK that 4 inmates were in a state of minimal depression and 4 had a moderate depression score. With the intervention the score was reduced from 9 (5) to 3 (3) (p <0.001).

Keywords: drug users, anxiety, depression, exercise.

\footnotetext{
${ }^{1}$ Laboratório de Avaliação e Intervenção em Fisioterapia. Centro Universitário de Anápolis, Anápolis, Goiás, Brasil.

${ }^{2}$ Colégio SESI Jundiai Anápolis, Goiás, Brasil.

* Autor correspondente: ftviviane@gmail.com
} 


\section{INTRODUÇÃO}

O consumo de substâncias psicoativas tem aumentado nos últimos anos, colocando em risco a saúde da população em geral, pois o uso de drogas em excesso causa a dependência e, consequentemente doenças, problemas psicossociais e prejuízos socioeconômicos, tornando- se, assim um problema de saúde pública (Prata \& Santos, 2009). O uso excessivo de tabaco e álcool está entre os dez fatores que mais causam doenças no mundo, e associado a outros problemas como pressão alta, colesterol traz danos reais à saúde com alto risco de morte (OMS, 2002).

O descontrole emocional causado pelo uso dessas drogas leva o indivíduo a buscar prazer maior em outros tipos de drogas (Silva \& Padilha, 2011). A falta da substância no organismo gera o que especialistas chamam de craving (desejo de repetir a experiência dos efeitos de uma dada substância) e esse desejo gerado pela abstinência provoca alterações comportamentais no indivíduo. Assim é essencial encontrar meios de intervenção que previna a dependência desde o início impedindo o agravamento da doença. E em casos mais avançados promover melhoras nas condições físicas, psicológica e social do indivíduo (OMS, 2001).

A intervenção deve levar em consideração o estado do dependente. Estudiosos tem mostrado que a prática de atividade física ajuda no combate de uma infinidade de doenças dos diversos sistemas do corpo humano, por liberar endorfina hormônio responsável pelo prazer, felicidade e satisfação e é uma alternativa para doenças como depressão e ansiedade, que comumente estão presentes no indivíduo que é dependente (Araújo et al., 2008). A educação física é considerada historicamente, como uma sabedoria de viver, ajuda no crescimento, desenvolvimento físico e mental, colaborando na formação do cidadão, por ser sinônimo de saúde considera-se que esteja compromissada com a existência humana (Satin, 1993; Babanti, 2012). E, por isso, pode auxiliar na recuperação do dependente.

Percebe-se então a importância de pesquisas que levem a um melhor entendimento sobre como a prática de atividades físicas podem contribuir para a recuperação e prevenção de recaídas de dependentes químicos. Assim, em virtude do aumento exagerado do consumo de drogas, principalmente pelo fato de não haver tratamento curativo, este estudo teve como objetivo verificar os efeitos da atividade física em caráter de lazer sobre os sintomas de ansiedade, depressão e da aptidão física de dependentes químicos em tratamento.

\section{MÉTODO}

Estudo experimental sem grupo de controle realizada no Centro de Reabilitação e Amparo Bom Jardim - Distrito de Interlândia - Anápolis Goiás. É um local que conta com: um campo de futebol society; uma quadra poliesportiva; uma piscina e uma grande área verde.

\section{Participantes}

O Centro atende uma população de 20 pessoas, no qual somente 10 concordaram por conveniência em participar da pesquisa. Foram incluídos os participantes com idade acima de 18 anos, do sexo masculino que tem em comum a dependência química (álcool, crack, maconha, cocaína entre outas substâncias psicoativas). Foram excluídos todos os pacientes que apresentaram condição clínica aguda que impossibilitaram a prática de exercicios, sem capacidade cognitiva ou em estado mental (lucidez momentanea) de responder o questionário. Todos os participantes assinaram o Termo de Consentimento Livre e Esclarecido (TCLE) e o estudo seguiu as orientações da Declaração de Helsinki e Resolução 466/12.

\section{Instrumentos e Procedimentos}

Após a assinatura do TCLE e o preenchimento de uma ficha de identificação com dados sobre idade, escolaridade e uso de medicamentos. Foram aplicados o questionário de BECK de ansiedade e depressão validados para população brasileira (Gomes-Oliveira et al., 2012). Em seguida, foram aferidos massa corporal $(\mathrm{kg})$ e estatura (m) utilizando uma balança digital (Filizola, modelo 2096 PP, São Paulo, Brasil) e um estadiômetro (Sanny, São Paulo, Brasil), realizado 5 minutos de aquecimento, os testes de 
abdominais e flexão de braço (Guedes \& Guedes, 2006). O teste de Cooper avaliou a aptidão cardiorrespiratória e a partir daí, foram feitas 12 intervenções, 2 vezes por semana por um período de 6 semanas (Guedes \& Guedes, 2006). No primeiro dia da semana foi utilizada uma academia artesanal montada nas dependências da instituição que contava com alguns equipamentos como barras, anilhas, alteres, banco e um aparelho de puxada. E no segundo dia ocorria a partida de futebol com 2 tempos de 25 minutos e intervalo de $10 \mathrm{~min}$.

\section{Análise estatística}

Os resultados foram expressos como média, desvio-padrão, frequências e porcentagens. Para verificar a normalidade dos dados será utilizado o teste de Shapiro-Wilk. A comparação pré e pósintervenção será realizado com o teste t-Student para variáveis com distribuição normal e teste de Wilcoxon para distribuição assimétrica. O nível de significância considerado será $<0,05$. Os dados serão analisados no software Statistical Package Social Science (SPSS).

\section{RESULTADOS}

Dos 10 pacientes que iniciaram o estudo 2 desistiram do tratamento. As características da amostra estão descritas na tabela 1 . Todos eram adultos jovens e 3 estavam com sobrepeso quando classificados de acordo com o IMC. Nenhum dos internos relatou fazer tratamento medicamentoso.

Tabela 1

Avaliação Inicial do grupo de estudo

\begin{tabular}{cc}
\hline Variáveis & Valor \\
\hline Idade (anos) & $29,38(5,63)$ \\
Massa corporal $(\mathrm{kg})$ & $78,72(11,91)$ \\
Estatura $(\mathrm{m})$ & $1,70(0,09)$ \\
IMC $\left(\mathrm{kg} / \mathrm{m}^{2}\right)$ & $27,31(3,94)$ \\
Escolaridade (f/\%) & \\
Ensino médio incompleto & $06(75)$ \\
Ensino médio completo & $02(25)$ \\
\hline
\end{tabular}

A tabela 2 descreve os resultados das variáveis de aptidão física e do questionário de BECK de ansiedade e depressão. Após a intervenção, os participantes realizaram maior número de flexões de membro superior $(p=0,03)$ e percorreu maior distância (200m) no teste de aptidão cardiorrespiratória $(p=0,002)$. Com relação ao número de abdominais 4 dos que iniciaram estavam abaixo da média esperada e 4 atingiram o número previsto de repetição.

Foi constado através do inventario de depressão de BECK que 4 internos se encontravam em estado de depressão mínima e 4 apresentavam score moderado de depressão. Com a intervenção foi reduzido o escore de 9 (5) para 3 (3) $(\mathrm{p}<0,001)$. Quanto à avaliação dos níveis de ansiedade verificou-se não houve diferença significativa.

Tabela 2

Comparação pré e pós intervenções dos testes de aptidão física e escores de ansiedade

\begin{tabular}{|c|c|c|c|}
\hline Variáveis & Pré-intervenção & Pós-intervenção & $\mathrm{p}$ \\
\hline Abdominais (n) & $28(12)$ & $30(12)$ & 0,13 \\
\hline $\begin{array}{l}\text { Flexões de } \\
\text { braço (n) }\end{array}$ & $16(7)$ & $18(8)$ & 0,03 \\
\hline $\begin{array}{l}\text { Teste de Cooper } \\
12 \text { min. (m) }\end{array}$ & $1718(577)$ & $1908(585)$ & 0,002 \\
\hline $\begin{array}{l}\text { Escore } \\
\text { depressão }\end{array}$ & $9(5)$ & $3(3)$ & $<0,001$ \\
\hline $\begin{array}{l}\text { Escore } \\
\text { ansiedade }\end{array}$ & $27(6)$ & $25(10)$ & 0,55 \\
\hline
\end{tabular}

\section{DISCUSSÃO}

Após o início da sessão de atividades físicas observou-se melhora significativa em duas das variáveis de aptidão física, flexão de braço e no teste de aptidão cardiorrespiratória. Foi observado, também importante melhora no score de depressão, inclusive a convivência e socialização do grupo dentro do Centro de reabilitação. Como o foco deste trabalho era observar como a prática de atividades físicas pode interferir de maneira significativa nas condições do reabilitando quanto à depressão e como coadjuvante na reabilitação e tratamento da dependência química, é importante considerar que o diálogo também foi parte importante do processo.

A aplicação dos questionários de BECK pré intervenção foi essencial para observar o quadro em que se encontrava a amostra em questão, tanto do estado depressivo quanto a variável ansiedade e, os testes físicos para se estimar a real condição física do reabilitando. Essa observação também foi feita em estudo anterior que estudou o auxílio do exercício físico foi constatado que é 
necessário observar o quadro de abstinência, o estado físico e psicológico em que se encontra o paciente, para então decidir quais atividades e em que intensidade pode ser desenvolvida, confirmando o que foi proposto e executado dentro deste estudo (Ferreira; Tufik; Mello, 2017).

Percebeu-se durante o estudo que, a abstinência favorece a ansiedade, e por ser complexo necessita de um estudo longitudinal já que demanda tempo para verificar as mudanças no estado comportamental do indivíduo, seja por efeitos da atividade física ou de qualquer outro tipo de intervenção. Já, a depressão pôde ser melhorada com as atividades físicas, pois estas promovem liberação de hormônios benéficos e foi percebido durante o presente estudo que houve diminuição no estado de angústia, raiva, tristeza e, consequentemente na depressão. Este fato pode ser confirmado por de outros estudos em que pesquisadores constataram que, no que diz ao lado emocional do indivíduo, a prática corporal (exercício) promove estímulos e mudanças no organismo, melhora no humor, raiva e confusão mental (Panda, 2011).

Percebeu-se também durante o protocolo de atividade física que, as atividades físicas aeróbias contribuíram com o estímulo físico, emocional e social da amostra. Isso pode ser confirmado através e outros estudos que afirmam que práticas de atividades físicas contribuem com os estímulos físico, social e emocional além das práticas frequentes de atividades físicas corroborarem com o processo de reabilitação de algumas doenças crônica (Mialick; Fracasso; Sahd, 2007; Coelho \& Burini, 2009). E, sendo a própria dependência química uma doença crônica pode favorecer na reabilitação do dependente.

Em um estudo realizado em um centro de atenção a dependência química constatou-se que, exercícios físicos aeróbios ou resistidos são auxiliadores na fase de recuperação do indivíduo (Caleffi, 2013). O presente estudo colabora com estes resultados, o autor enfatiza sobre a necessidade de um profissional de educação física para coordenar e orientar o modo como são realizadas as atividades, como forma de prevenção a possíveis danos à integridade física do reabilitando.
Ao observar o desempenho dos voluntários nos exercícios por meio dos testes notou-se uma melhora no estado de humor pós intervenções, principalmente após as partidas de futebol, estima-se que tenha elevando também à autoestima, melhorado a coordenação motora, além de ajudar nos distúrbios de sono. O que vai de encontro a estudos anteriores onde os pesquisadores afirmam terem encontrado interferência significativa do exercício físico nos fatores emocional, cognitivo e motor (Ferreira, 2016).

Entende-se em razão das variáveis encontradas neste estudo ter havido resposta positiva através dos instrumentos utilizados no desenvolvimento desta pesquisa. Hoje, se sabe que a depressão é uma patologia de proporções mundiais, que atinge todos os níveis da sociedade e quando aliada a altos níveis de ansiedade advindos de dependência química torna-se um distúrbio ainda mais grave. A ansiedade é um fator que desencadeia diversas formas de desequilíbrio na estrutura metabólica e psicológica do ser humano, e requer uma avaliação mais detalhada. Por esse motivo demanda cuidados de vários profissionais, como médicos, psicólogos, líderes religiosos, profissionais de educação física, familiares e a sociedade como um todo.

\section{CONCLUSÕES}

O resultado obtido com este trabalho nos leva a acreditar que a intervenção de um profissional de educação física ao introduzir a prática de exercícios físicos como coadjuvante aliada aos demais tratamentos pode se tornar uma importante ferramenta na reabilitação da dependência, prevenção de recaídas e na manutenção dos níveis normais de ansiedade e depressão. Pode proporcionar ao indivíduo bemestar, qualidade de vida e reinserção a sociedade e quem sabe preservando-o longe do mundo das drogas. Devido ao curto espaço de tempo do estudo entendemos que há necessidade de mais estudos para que se possa refletir melhor sobre este tema, que tanto aflige os que lutam contra seus malefícios, que causa também sofrimento de seus familiares. 
Conclui-se com este estudo que atividade física tem influência benéfica no tratamento da dependência, principalmente, nas fases de recuperação da abstinência e reintegração social do adicto. Todavia quanto à cronicidade da patologia em questão há necessidade de mais estudos, principalmente, de longo prazo, para que venha contribuir de maneira contundente com a eficácia do tratamento. Salienta-se ainda a importância da participação dos familiares e profissionais envolvidos no processo de reabilitação.

Agradecimentos:
Laboratório da Avaliação e Intervenção em
Fisioterapia-LAIF.

\section{Conflito de Interesses:}

Nada a declarar.

\section{Financiamento:}

Nada a declarar.

\section{REFERÊNCIAS}

Araújo, R. B., Oliveira M. S., Pedroso, R. S., Miguel, A. C., \& Castro, M. G. T. (2008). Craving e dependência química: conceito, avaliação e tratamento: Craving and chemical dependence: concept, evaluation and treatment. Jornal Brasileiro de Psquiatria, 57(1), 57-63. doi: 10.1590/S004720852008000100011

Barbanti, E. J. (2012). A importância do exercício físico no tratamento da dependência química. Educação Física em Revista, 6(1), 1-9.

Caleffi, R. P. (2013). Educação Física e a Reabilitação de Usuários de Álcool e outras drogas. Monografia apresentada a Universidade Federal de Goiás.

Coelho, C. F., \& Burini, R. C. (2009). Atividade física para prevenção e tratamento das doenças crônicas não transmissíveis e da incapacidade funcional. Revista de Nutrição, 22(6), 937-946. doi: 10.1590/S1415-52732009000600015

Ferreira, S. E., Tufik, S., \& Mello, M. T (2001). Neuroadaptação: uma proposta alternativa de atividade física para usuários de drogas em recuperação. Revista Brasileira de Ciências do Esporte, 9(1), 31-39.

Ferreira, S. E., Santos, A. K. M., Okano, A. H., Gonçalves, B. S. B., \& Araújo, J. F. (2017). Efeitos agudos do exercício físico no tratamento da dependência química. Revista Brasileira Ciências Esporte, 39(2), 123-131. doi: 10.1016/j.rbce.2016.01.016

Gomes-Oliveria, M. H., Gorestein, C., Neto, F. L., Andrade, L. H., \& Wang, Y. P. (2012). Validation of the Brazilian Portuguese version of the Beck Depression Inventory-II in a community sample. Revista Brasileira de Psiquiatria, 34(4), 389-394. doi: 10.1016/j.rbp.2012.03.005

Guedes, D. P, \& Guedes, J. E. R. P. (2006). Manual Prático Para Avaliação Em Educação Física. Barueri: Manole.

Mialick, E. S., Fracasso, L., \& Sahd, S. M. P. V. (2007). A importância da pratica da atividade física como auxilio no processo de tratamento para a dependência química em pessoas de 18 a 35 anos. VI Concurso Nacional de Monografias sobre o tema Drogas para Universitários 2006/2007, promovido pelo Centro de Integração Empresa Escola - CIEE, em parceria com a Secretaria Nacional Antidrogas SENAD.

Organização Mundial da Saúde. (2001). Transtornos devido ao uso de substâncias. Em Organização PanAmericana da Saúde \& Organização Mundial da Saúde (Orgs.). Relatório sobre a saúde no mundo. Saúde Mental: nova concepção.

Panda, M. D. J. (2011). A influência de um programa de exercícios físicos no estado emocional dos indivíduos cadastrados no esf/primavera. Revista Biomotriz, 5.

Pereira, G. S., \& Gomes, M. F. L. (2015). As consequências da dependência de substancias psicoativas no cotidiano dos(as) usuários(as) na casa de acolhida adulto: o posicionamento da equipe psicossocial. Resumo expandido: VII Jornada Internacional de Políticas Públicas. Universidade Federal do Maranhão.

Pratta, E. M. M., \& Santos, M. A. (2009). O Processo Saúde-Doença e a Dependência Química: Interfaces e Evolução Revista Psicologia Teoria e Prática, 25(2), 203-211.

Silva, S. E. D., \& Padilha, M. I. (2011). Atitudes e comportamentos de adolescentes em relação à ingestão de bebidas alcoólicas. Revista da Escola de Enfermagem, 45(5), 1063-1069.

World Health Organization. (2002). Reducing Risk, Promoting Health Life. World Health Report. Geneva, Switzerland. 